TRANSACTIONS OF THE

AMERICAN MATHEMATICAL SOCIETY

Volume 350, Number 9, September 1998, Pages 3707-3716

S $0002-9947(98) 02062-5$

\title{
HYPERCYCLICITY IN THE SCATTERING THEORY FOR LINEAR TRANSPORT EQUATION
}

\author{
H. EMAMIRAD
}

\begin{abstract}
We show how the hypercyclicity of the transport semigroup can intervene in the scattering theory to characterize the density property of the Lax and Phillips representation theorem and conversely, how the existence of the wave operators of the scattering theory can be used for recovering the hypercyclicity of the absorbing transport group in some weighted $L^{1}$ spaces.
\end{abstract}

\section{INTRODUCTION}

The study of the scattering theory for transport phenomena was initiated by Lax and Phillips [L-P1] for the streaming free group

$$
U_{0}(t) f(x, v)=f(x-t v, v),
$$

in the context of the Hilbert space $X=L^{2}\left(\mathbb{R}^{3} \times S^{2}\right)$.

From a compact convex subset $\Omega$ of $\mathbb{R}^{3}$ they defined the incoming and outgoing subspaces $D_{-}$and $D_{+}$as follows : Let $\rho$ be the radius of a ball $B_{\rho}$ around the origin which contains all points of $\Omega$. Let $E_{+}=\{(x, v) \mid x \cdot v \geq \rho\}$ be the free forward and $E_{-}=\{(x, v) \mid x \cdot v \leq-\rho\}$ be the free backward point sets of $\mathbb{R}^{3} \times S^{2}$. Corresponding to these sets they defined

$$
D_{ \pm}=\left\{f \in L^{2}\left(\mathbb{R}^{3} \times S^{2}\right) \mid \operatorname{supp} f \subset E_{ \pm}\right\}
$$

and they have proved that for the dynamics $U(t)=U_{0}(t)$, the following representation theorem holds (see also [L-P2]).

Theorem 1.1. The subspaces $D_{+}$and $D_{-}$satisfy the following properties:

$(i)_{+} U(t) D_{+} \subset D_{+} \quad$ for $\quad t \geq 0$

$(i)_{-} U(t) D_{-} \subset D_{-}$for $t \leq 0$

(ii) $\bigcap_{t \in \mathbb{R}} U(t) D_{ \pm}=\{0\}$,

(iii) $\bigcup_{t \in \mathbb{R}} U(t) D_{ \pm} \quad$ is dense in $X$.

In [Em1] and [Em2], the author generalized the above representation theorem in the Banach space $X=L^{1}\left(\mathbb{R}^{n} \times \mathcal{V}\right)$ for the transport semigroup $U(t)$, which is governed by the transport equation

$$
\frac{\partial u}{\partial t}=-v \cdot \nabla_{x} u-\sigma_{a}(x, v) u+\int_{\mathcal{V}} k\left(x, v^{\prime}, v\right) u\left(x, v^{\prime}, t\right) d v^{\prime},
$$

Received by the editors October 29, 1996.

1991 Mathematics Subject Classification. Primary 47D05; Secondary 82A70.

Key words and phrases. Hypercyclic semigroup, Lax and Phillips scattering theory, two similar semigroups.

(C)1998 American Mathematical Society 
where

$$
\mathcal{V}=\left\{v \in \mathbb{R}^{n}\left|0<v_{\min } \leq\right| v \mid \leq 1\right\}
$$

is the velocity space; the absorbing cross section $\sigma_{a}(x, v)$ and producing source function $k\left(x, v, v^{\prime}\right)$ are the positive functions in $L^{\infty}\left(\mathbb{R}^{n} \times \mathcal{V}\right)$ and $L^{\infty}\left(\mathbb{R}^{n} \times \mathcal{V} \times \mathcal{V}\right)$ having their supports as functions of $x$ in a compact convex subset $\Omega$ of $\mathbb{R}^{n}$. By assuming the transparent boundary condition on (1.2) $U(t)$ becomes a strongly continuous group for which Theorem 1.1 holds.

In all the applications of the Lax and Phillips representation theorem the most difficult point is the density property (iii). Since for $t \geq 0, U(t) D_{+} \subset D_{+}$, thus

$$
\overline{\bigcup_{t \in \mathbb{R}} U(t) D_{+}}=\overline{\bigcup_{t \leq 0} U(t) D_{+}}=X \text {. }
$$

Similarly,

$$
\overline{\bigcup_{t \in \mathbb{R}} U(t) D_{-}}=\overline{\bigcup_{t \geq 0} U(t) D_{-}}=X .
$$

Here a crucial question arises: is it possible to characterize a subset of the incoming or outgoing subspaces $D_{ \pm}$which generates this dense subset? It seems that this question is closely related to the hypercyclic character of the semigroups $V_{ \pm}(t)$ which are the restrictions of $U(t)$ to $D_{ \pm}$for $\mp t \geq 0$.

A systematic study of a hypercyclic strongly continuous $\left(C_{0^{-}}\right)$semigroup was recently initiated in [D-S-W]. A $C_{0}$-semigroup of bounded linear oprators $\{T(t)\}_{t \geq 0}$ is called hypercyclic provided that there exists $x \in X$ such that $\{T(t) x \mid t \geq 0\}$ is dense in $X$.

In the next section we will prove that on $X=L_{\omega_{ \pm}}^{1}\left(E_{ \pm}\right)$, where $\omega_{ \pm}(x, v)>0$ is an adequate weight function, the semigroups $V_{0}^{ \pm}(t)=\chi_{ \pm} U_{0}(\mp t)$, where $\chi_{ \pm}$are the characteristic functions of $E_{ \pm}$and $U_{0}(t)$ is defined in (1.1), are hypercyclic. By means of this result we retrieve the Lax and Phillips representation theorem in $L_{\omega}^{1}\left(\mathbb{R}^{n} \times \mathcal{V}\right)$ by proving that the dense subset in (iii) can be generated by a single element of $X$. Then, we extend the weight functions $\omega_{ \pm}$on the whole $\mathbb{R}^{n} \times \mathcal{V}$ in such a manner that by imposing the classical assumptions on the pair $\left(k, \sigma_{a}\right)$ appearing in (1.2), $U(t)$ becomes a $C_{0}$-group on $L_{\omega}^{1}\left(\mathbb{R}^{n} \times \mathcal{V}\right)$. By defining $V^{ \pm}(t)=\chi_{ \pm} U(\mp t)$, we prove that $V^{ \pm}(t)=V_{0}^{ \pm}(t)$, which in turn establishes the Lax and Phillips representation theorem for $U(t)$. This result is already announced in [Em3]. In the section 3 , we prove that if $\{S(t)\}_{t \geq 0}$ and $\{T(t)\}_{t \geq 0}$ are two similar $C_{0}$-semigroups and if one of them is hypercyclic, then the other one is also hypercyclic. In the case when $\{S(t)\}_{t \in \mathbb{R}}$ and $\{T(t)\}_{t \in \mathbb{R}}$ are two $C_{0}$-groups we show that how the existence of the wave operators in scattering theory can help us to establish the similarity between these groups.

In the fourth section we will prove the hypercyclicity of the streaming free group $U_{0}(t)$ in some $L_{\omega}^{1}\left(\mathbb{R}^{n} \times \mathcal{V}\right)$. We show that, for any $\alpha>0$, there exists a weight function $\omega$ such that

$$
\left\|U_{0}(t)\right\|_{L^{1}\left(\mathbb{R}^{n} \times \mathcal{V}\right)} \leq e^{\alpha|t|} \quad \text { for all } t \in \mathbb{R} .
$$

The construction of this weight function is similar to those of [D-S-W] for translation groups.

Unfortunately with an estimation like (1.5) we are not able to use the existence theorems (as Cook's Lemma [R-S] or Theorem 1 of [Um]) of the wave operators 
in order to obtain the hypercyclicity of the transport group $U(t)$. In fact, in any abstract result for the existence of wave operators the free dynamic should be uniformly bounded in the underlying Banach space.

However, if the kernel $k$ is identically zero, the equation (1.2) generates a $C_{0^{-}}$ group $U_{1}(t)$ which is called absorbing transport group. In this case the wave operators have an explicit form and we don't need to use an abstract theorem for their existence. By the way, a particular case of this equation (when $\sigma_{a}(x, v)=\sigma(x)$ ) and the corresponding scattering operator are used in the inverse problems in computerized tomography (see $[$ Em-A]).

\section{HyperCyClicity of the transport SEMigroup}

Let $\mathcal{V}$ be the velocity space defined in (1.3) and $E$ be a subset of $\mathbb{R}^{n} \times \mathcal{V}$.

Definition 2.1. By an admissible weight function on $E$ we mean a measurable positive function $\omega$ satisfying

$$
\omega(x-t v, v) \leq r(t) \omega(x, v)
$$

for all $(x, v) \in E$, where $r$ is a positive function such that $\liminf _{t \rightarrow \infty} r(t)=0$.

A prototype example of an admissible weight function is

$$
\omega(x, v)=\exp (x \cdot v) .
$$

Let us denote by $L_{\omega}^{1}(E)$ the space of measurable functions $f: E \longmapsto \mathbb{C}$ such that

$$
\|f\|_{E}=\int_{E}|f(x, v)| \omega(x, v) d x d v<\infty .
$$

Let $\omega_{+}$and $\omega_{-}$be two admissible weight functions defined respectively on $E_{+}$ and $E_{-}$. Let $\omega$ be a positive extension of $\omega_{ \pm}$on $\mathbb{R}^{n} \times \mathcal{V}$. On $L_{\omega}^{1}\left(\mathbb{R}^{n} \times \mathcal{V}\right)$ we will define the streaming free group $\left\{U_{0}(t)\right\}_{t \in \mathbb{R}}$ as in (1.1), which in turn defines the dynamics $V_{0}^{ \pm}(t)=\chi_{ \pm} U_{0}(\mp t)$ on $L_{\omega_{ \pm}}^{1}\left(E_{ \pm}\right)$.

Theorem 2.2. $\left\{V_{0}^{ \pm}(t)\right\}_{t \geq 0}$ are strongly continuous hypercyclic semigroups on $L_{\omega_{ \pm}}^{1}\left(E_{ \pm}\right)$.

For the proof of this theorem we need the following lemma.

Lemma 2.3 [D-S-W, Theorem 2.3]. Let $\{T(t)\}_{t \geq 0}$ be a $C_{0}$-semigroup on a Banach space $X$. Let $X_{0}=\left\{x \in X \mid \lim _{t \rightarrow \infty} T(t) x=0\right\}$ and $X_{\infty}=\{x \in X \mid \forall \epsilon>$ $0, \exists u \in X$ and $t>0$ such that $\|u\|<\epsilon$ and $\|T(t) u-x\|<\epsilon\}$. If both $X_{0}$ and $X_{\infty}$ are dense subsets of $X$, then $\{T(t)\}_{t \geq 0}$ is hypercyclic.

Proof of Theorem 2.2. The fact that $V_{0}^{ \pm}(t)$ are two $C_{0}$-semigroups can be proved by the standard argument of translation semigroups. Here we will prove the hypercyclicity of $V_{0}^{-}(t)$; the same argument will work for $V_{0}^{+}(t)$.

Let $f$ be an element of $L_{\omega_{-}}^{1}\left(E_{-}\right)$with compact support in $E_{-}$. Thus, there is some $R>\rho$ such that for any $(x, v) \in \mathbb{R}^{n} \times \mathcal{V}$ with $-R \geq x \cdot v, f(x, v)=0$. Therefore for any $(x, v) \in E_{-}$and $t \geq(R-\rho) / v_{\min }^{2}$ one has $x \cdot v-t|v|^{2} \leq-\rho-t v_{\min }^{2} \leq-R$ and $f(x-t v, v)=0$. This implies that $X_{0}$ contains all functions of compact support in $L_{\omega_{-}}^{1}\left(E_{-}\right)$, so that $X_{0}$ is dense. 
For the density of $X_{\infty}$, we choose an arbitrary function $f$ of compact support in $E_{-}$and we define $u_{t}(x, v)=f\left(\tau_{t}(x, v), v\right)$, where $\tau_{t}(x, v)=(x+t v, v)$. For any $t \geq 0$ and $u_{t} \in L_{\omega_{-}}^{1}\left(E_{-}\right)$, we have

$$
\begin{aligned}
\left\|u_{t}\right\|_{E_{-}} & =\int_{E_{-}}|f(x+t v, v)| \omega_{-}(x, v) d x d v \\
& =\int_{E_{-}}|f(x, v)| \omega_{-}(x-t v, v) d x d v \\
& \leq r_{-}(t)\|f\|_{E_{-}} .
\end{aligned}
$$

Hence, from the vanishing property of $r_{-}(t)$, for any $\epsilon>0$, we have $\left\|u_{t}\right\|_{E_{-}}<\epsilon$ for some $t$ enough large, while $V_{0}^{-}(t) u_{t}=f$. Consequently $f \in X_{\infty}$. Since $f$ has been picked from a dense subset, $X_{\infty}$ is dense in $L_{\omega_{-}}^{1}\left(E_{-}\right)$and Lemma 2.3 concludes.

Corollary 2.4. The Lax and Phillips representation theorem holds for the streaming free group $U_{0}(t)$ in $L_{\omega}^{1}\left(\mathbb{R}^{n} \times \mathcal{V}\right)$.

Proof. The properties (i) and (ii) can be obtained readily as in [Em1]. But for (iii) we have even more; in fact, we will prove that there exists $\varphi_{+} \in L_{\omega_{+}}^{1}\left(E_{+}\right)$such that instead of $\left(1.4^{+}\right)$we have

$$
\overline{\bigcup_{t \leq 0} U_{0}(t) D_{+}}=\overline{\bigcup_{t \leq 0, s \geq 0} U_{0}(t) V_{0}^{+}(s)\left\{\varphi_{+}\right\}}=X .
$$

In fact for any $\epsilon>0$ and any $f \in L_{\omega}^{1}\left(\mathbb{R}^{n} \times \mathcal{V}\right)$ there exists $g \in \mathcal{C}_{0}^{\infty}\left(\mathbb{R}^{n} \times \mathcal{V}\right)$ such that $\|f-g\|_{\mathbb{R}^{n} \times \mathcal{V}}<\frac{\epsilon}{2}$. Let $t$ be enough large such that $h=U_{0}(t) g \in L_{\omega_{+}}^{1}\left(E_{+}\right)=$ $D_{+}$. From the hypercyclicity of $V_{0}^{+}(s)$ it follows that there exists $s \geq 0$ such that $\left\|V_{0}^{+}(s) \varphi_{+}-h\right\|_{E_{+}}<\epsilon\left(r_{+}(t)+r_{-}(t)\right)^{-1} / 2$, for some $\varphi_{+}$. Hence,

$$
\begin{gathered}
\left\|f-U_{0}(-t) V_{0}^{+}(s) \varphi_{+}\right\|_{\mathbb{R}^{n} \times \mathcal{V}} \leq \frac{\epsilon}{2}+\left\|g-U_{0}(-t) V_{0}^{+}(s) \varphi_{+}\right\|_{\mathbb{R}^{n} \times \mathcal{V}} \\
\leq \frac{\epsilon}{2}+\left(r_{+}(t)+r_{-}(t)\right)\left\|U_{0}(t) g-V_{0}^{+}(s) \varphi_{+}\right\|_{\mathbb{R}^{n} \times \mathcal{V}} \leq \epsilon .
\end{gathered}
$$

The same argument holds for $E_{-}$and we can write

$$
\overline{\bigcup_{t \geq 0} U_{0}(t) D_{-}}=\overline{\bigcup_{t \geq 0, s \geq 0} U_{0}(t) V_{0}^{-}(s)\left\{\varphi_{-}\right\}}=X
$$

We will now describe some condition on the pair $\left(k, \sigma_{a}\right)$, appearing in $(1.2)$, for the strong continuity of the transport semigroup. We assume that a finite convex scattering body $\Omega$ is surrounded by a vacuum and we define the incoming and outgoing spaces as before. Let $\omega_{ \pm}$be an admissible weight function on $E_{ \pm}$. Define

$$
\omega(x, v)= \begin{cases}\omega_{+}(x, v) \quad \text { if } \quad(x, v) \in E_{+}, \\ \omega_{-}(x, v) \quad \text { if } \quad(x, v) \in E_{-}, \\ 1 \quad \text { elsewhere. }\end{cases}
$$

As in $[\mathrm{Si}]$, we assume that the pair $\left(k, \sigma_{a}\right)$ satisfies the following hypotheses: 
(H1) $k\left(x, v, v^{\prime}\right)$ is a nonnegative measurable function on $\mathbb{R}^{n} \times V \times V$ for Lebesgue measure $d x d v d v^{\prime}$ and $\sigma_{a}(x, v)$ is a nonnegative measurable function on $\mathbb{R}^{n} \times V$ for Lebesgue measure $d x d v$.

(H2) For each $(x, v), k(x, v,$.$) is in L^{1}(V)$.

(H3) $\sigma_{a}$ and $\sigma_{p} \equiv \int_{V} k\left(x, v, v^{\prime}\right) d v^{\prime}$ are uniformly bounded on $\mathbb{R}^{n} \times V$.

(H4) $k\left(x, v, v^{\prime}\right)$ and $\sigma_{a}(x, v)$ vanish for $x \notin \Omega$.

We consider the following Cauchy problem in $L_{\omega}^{1}\left(\mathbb{R}^{3} \times \mathcal{V}\right)$ :

$$
\left\{\begin{array}{l}
\frac{\partial u}{\partial t}=T u, \\
u(x, v, 0)=f(x, v),
\end{array}\right.
$$

where $T=T_{0}+A=T_{0}+A_{a}+A_{p}$ with $T_{0} f \equiv-v \cdot \nabla_{x} f, A_{a} f \equiv-\sigma_{a}(x, v) f$ and $\left[A_{p} f\right](x, v) \equiv \int_{\mathcal{V}} k\left(x, v^{\prime}, v\right) f\left(x, v^{\prime}\right) d v^{\prime}$.

Theorem 2.5. Under hypotheses (H1) to (H4), the operator $T$ generates a $C_{0}-$ group on $L_{\omega}^{1}\left(\mathbb{R}^{3} \times \mathcal{V}\right)$.

Proof. Knowing that $T_{0}$ generates a $C_{0}$-group $U_{0}(t)$, it is enough to show that $A$ is a bounded perturbation of $T_{0}$. According to (H3), the uniform boundedness of $\sigma_{a}$ on $\mathbb{R}^{n} \times \mathcal{V}$ implies the boundedness of the operator $A_{a}$. While for the boundedness of $A_{p}$, we have to prove that

$$
\int_{\mathcal{V}} k\left(x, v^{\prime}, v\right) \omega(x, v) d v \leq C \omega\left(x, v^{\prime}\right) \quad \text { for all } \quad\left(x, v^{\prime}\right) \in \mathbb{R}^{n} \times \mathcal{V} .
$$

Since for $(x, v) \in E_{ \pm}$we have $|x| \geq|x||v| \geq \pm x \cdot v \geq \rho$. Thus (H4) implies that for $x \notin \Omega$, the left-hand side of (2.2) is identically zero and for $x \in \Omega, \omega(x, v)=1$; hence (2.2) follows from (H2).

Let us denote by $U(t)$ the $C_{0}$-group generated by $T$. Define $V^{ \pm}(t)=\chi_{ \pm} U(\mp t)$ on $L_{\omega_{ \pm}}^{1}\left(E_{ \pm}\right)$.

Theorem 2.6. $V^{ \pm}(t)=V_{0}^{ \pm}(t)$ on $L_{\omega_{ \pm}}^{1}\left(E_{ \pm}\right)$.

Proof. According to Duhamel's formula

$$
U(-t)=U_{0}(-t)-\int_{-t}^{0} U_{0}(-t-s) A U(s) d s
$$

or

$$
V^{+}(t)=V_{0}^{+}(t)-\chi_{+} \int_{0}^{t} U_{0}(-\tau) A U(\tau-t) d \tau
$$

Taking (H4) into account, for any $f$, the function $g(x, v)=[A U(\tau-t) f](x, v)$ vanishes for $x \notin \Omega$. Since for any $(x, v) \in E_{+}$one has $(x+\tau v, v) \in E_{+}$, thus $\chi_{+}(x, v) U_{0}(-\tau) g(x, v)=0$ for all $\tau \geq 0$. This together with (2.3) implies that $V^{+}(t)=V_{0}^{+}(t)$ and the same argument holds for $V^{-}(t)=V_{0}^{-}(t)$.

Analogously to Corollary 2.4, we can show that

Corollary 2.7. The Lax and Phillips representation theorem holds for the transport group $U(t)$ in $L_{\omega}^{1}\left(\mathbb{R}^{n} \times \mathcal{V}\right)$. 
Proof. By virtue of Corollary 2.4, it is enough to remark that $U(t) D_{ \pm} \subset D_{ \pm}$for $\pm t \geq 0$. In fact, $U(t) f=U_{0}(t) f+\int_{0}^{t} U(t-s) A U_{0}(s) f d s$. For $f \in D_{+}, U_{0}(s) f \in D_{+}$ and $A U_{0}(s) f$ is identically zero. Moreover, we have

$$
\overline{\bigcup_{ \pm t \geq 0} U(t) D_{\mp}}=\overline{\bigcup_{ \pm t \geq 0, s \geq 0} U(t) V^{\mp}(s)\left\{\varphi_{\mp}\right\}}=X .
$$

\section{The Similarity Between two $C_{0}$-SEMigroups}

In this section we introduce the notion of similarity between two $C_{0}$-semigroups and we give a sufficient condition to realize this similarity for two $C_{0}$-groups.

Definition 3.1. Let $\{S(t)\}_{t \geq 0}$ and $\{T(t)\}_{t \geq 0}$ be two $C_{0}$ - semigroups on a Banach space $X . S(t)$ and $T(t)$ are called similar if there exists an isomorphism $P$ on $X$ such that

$$
T(t)=P^{-1} S(t) P \quad \text { for all } t \geq 0 .
$$

Theorem 3.2. Let $S(t)$ and $T(t)$ be two similar $C_{0}$-semigroups. If one of them is hypercyclic, then the other one is also hypercyclic.

Proof. Let $S(t)$ be a hypercyclic $C_{0}$-semigroup and $y$ an arbitrary element of $X$. For $x=P y$ and $\epsilon>0$, there exist $x_{0} \in X$ and $t \geq 0$ such that $\left\|S(t) x_{0}-x\right\| \leq \epsilon M^{-1}$, where $M=\left\|P^{-1}\right\|$. Hence, for $y_{0}=P^{-1} x_{0}$ we have

$$
\left\|T(t) y_{0}-y\right\| \leq M\left\|S(t) P y_{0}-P y\right\|<\epsilon,
$$

which proves the hypercyclicity of $T(t)$.

One of the essential aims in the scattering theory is to establish the similarity for a pair of linear operators. Consider a pair of linear operators $T$ and $T_{0}$, where $T$ is a bounded perturbation of $T_{0}\left(T=T_{0}+A\right.$ with $\left.A \in \mathcal{L}(X)\right)$. Assume that both $T$ and $T_{0}$ generate the bounded $C_{0}$-groups $e^{t T}$ and $e^{t T_{0}}$ on $X$. Then the existence of the wave operators (see $[\mathrm{Em} 2],[\mathrm{Um}]$ )

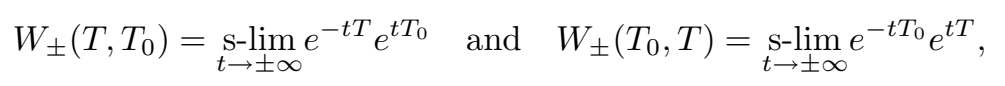

implies that

$$
W_{ \pm}\left(T, T_{0}\right) W_{ \pm}\left(T_{0}, T\right) f=W_{ \pm}\left(T_{0}, T\right) W_{ \pm}\left(T, T_{0}\right) f=f \quad \text { for all } \quad f \in X,
$$

and by letting $s \rightarrow \pm \infty$ in

$$
e^{t T} e^{-s T} e^{s T_{0}}=e^{(t-s) T} e^{(s-t) T_{0}} e^{t T_{0}}
$$

we obtain the following intertwining identity:

$$
e^{t T} W_{ \pm}\left(T, T_{0}\right) f=W_{ \pm}\left(T, T_{0}\right) e^{t T_{0}} f, \quad \text { for all } \quad f \in X .
$$

This proves that the $C_{0}$-groups $e^{t T}$ and $e^{t T_{0}}$ (consequently $T$ and $T_{0}$ ) are mutually similar. 


\section{HyPERCYCLICITY OF THE TRANSPORT ABSORBING GROUP}

For the characterization of the hypercyclic streaming free group $U_{0}(t) f(x, v)=$ $f(x-t v, v)$, we need the following lemma.

Lemma 4.1 [D-S-W, Theorem 2.2 and Theorem 2.5]. Let $\{T(t)\}_{t \geq 0}$ be a $C_{0}$-group on a Banach space $X$. Then the following assertions are equivalent:

(i) The semigroup $\{T(t) \mid t \geq 0\}$ is hypercyclic.

(ii) The semigroup $\{T(-t) \mid t \geq 0\}$ is hypercyclic.

(iii) For all $\epsilon>0$ there exists a dense subset $D \subset X$ such that for all $f \in D$ there exists a dense subset $D^{\prime} \subset X$ such that for all $g \in D^{\prime}$ there exist $h \in X$ and $t>0$ such that $\|g-h\|<\epsilon$ and $\|f-T(t) h\|<\epsilon$.

Throughout this section, we designate by $\|\cdot\|_{\omega}$ the norm of $L_{\omega}^{1}\left(\mathbb{R}^{n} \times \mathcal{V}\right)$, where $\omega$ is an admissible weight function.

Theorem 4.2. For any $\alpha>0$ there exists an admissible weight function $\omega(x, v)$ on $\mathbb{R}^{n} \times \mathcal{V}$ such that the steaming free group $\left\{U_{0}(t) \mid t \in \mathbb{R}\right\}$ is hypercyclic in $L_{\omega}^{1}\left(\mathbb{R}^{n} \times \mathcal{V}\right)$ and satisfies

$$
\left\|U_{0}(t) f\right\|_{\omega} \leq e^{\alpha|t|}\|f\|_{\omega} \quad \text { for all } t \in \mathbb{R} \text { and } f \in L_{\omega}^{1}\left(\mathbb{R}^{n} \times \mathcal{V}\right) .
$$

For the proof of this theorem, we need to construct a weight function $\omega$ such that it is admissible in some sense for $t \rightarrow \pm \infty$. We proceed with this construction in the following lemma.

Lemma 4.3 . For any $\alpha>0$ there exists a weight function $\omega$ such that for any compact set $K \subset \mathbb{R}^{n}$ there exists a positive function $r$ such that $\lim _{\text {inf }} \rightarrow \pm \infty r(t)=0$ and

$$
\omega(x-t v, v) \leq r(t) \omega(x, v) \quad \text { for all }(x, v) \in K \times \mathcal{V} \text { and all } t \in \mathbb{R}
$$

Furthermore

$$
\frac{\omega(x+t v, v)}{\omega(x, v)} \leq e^{\alpha|t|} \quad \text { for all }(x, v) \in \mathbb{R}^{n} \times \mathcal{V} \text { and all } t \in \mathbb{R}
$$

Proof. For any $\alpha>0$, we can choose an even real-valued function $\phi: \mathbb{R} \longmapsto \mathbb{R}$ such that $\phi$ is continuously differentiable, its derivative is bounded by $\alpha$ and $\phi(t)=0$ for all $t \in[-\rho, \rho]$ and

$$
\begin{aligned}
& \limsup _{s \rightarrow \infty} \phi(s)=\limsup _{s \rightarrow-\infty} \phi(s)=\infty \\
& \liminf _{s \rightarrow \infty} \phi(s)=\liminf _{s \rightarrow-\infty} \phi(s)=-\infty
\end{aligned}
$$

For example the following characterization of its derivative can determine entirely this function: Take an even function $\phi \in \mathcal{C}^{1}(\mathbb{R})$ such that $\left|\phi^{\prime}\right| \leq \alpha$ and

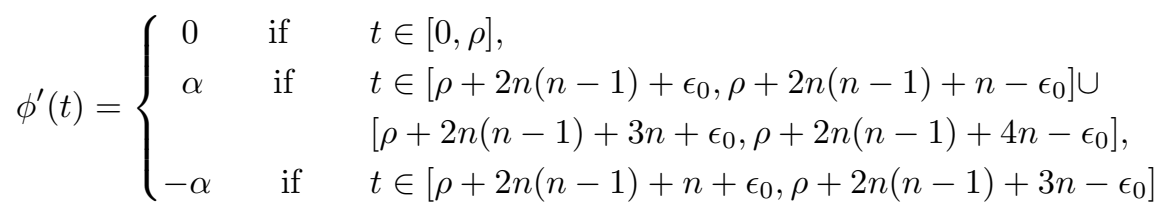

for $n=1,2, \cdots$ and some $\epsilon_{0}>0$ small enough. 
Let us define the weight function $\omega(x, v)=e^{\phi(x \cdot v)}$. Then

$$
\frac{\omega(x-t v, v)}{\omega(x, v)} \leq \exp \left\{-\int_{0}^{t} \phi^{\prime}\left(x \cdot v-s|v|^{2}\right)|v|^{2} d s\right\} .
$$

Since $|v| \leq 1$ and bounded away from zero, for any $(x, v) \in K \times \mathcal{V}$ we have $|x \cdot v| \leq M$ and there exists $a \in \mathbb{R}$ such that

$$
\exp \left\{-\int_{0}^{t} \phi^{\prime}\left(x \cdot v-s|v|^{2}\right)|v|^{2} d s\right\} \leq \exp \left\{\alpha n+\int_{a}^{a-t|v|^{2}} \phi^{\prime}(s) d s\right\}=r(t),
$$

where $n$ is the largest integer such that $\rho+2 n(n-1) \leq M$. The fact that for any $a \in \mathbb{R}$

$$
\limsup _{t \rightarrow \pm \infty} \int_{a}^{t} \phi^{\prime}(s) d s=+\infty \quad \text { and } \quad \liminf _{t \rightarrow \pm \infty} \int_{a}^{t} \phi^{\prime}(s) d s=-\infty
$$

implies (4.2) and by (4.4) we infer easily (4.3).

Proof of Theorem 4.2. Let $\omega$ be the weight function constructed in the previous lemma. Let $f$ and $g$ be two functions in $L_{\omega}^{1}\left(\mathbb{R}^{n} \times \mathcal{V}\right)$ with compact support, say $K \times \mathcal{V}$. We will show that Condition (iii) of Lemma 4.1 holds with $f$ and $g$. Since these functions are taken from a dense set, this implies that $\left\{U_{0}(t) \quad t \geq 0\right\}$ is hypercyclic. Since the weight function is even, we conclude that $\left\{U_{0}(t) t \leq 0\right\}$ is also a hypercyclic semigroup on $L_{\omega}^{1}\left(\mathbb{R}^{n} \times \mathcal{V}\right)$ which in turn proves the theorem.

By the notation in the proof of Theorem 2.2, $\tau_{t}(x, v)=(x+t v, v)$, we can choose $t$ large enough so that $\tau_{t}(K \times \mathcal{V}) \cap K \times \mathcal{V}=\emptyset$ and define

$$
h(x, v)= \begin{cases}g(x, v) & \text { if } \quad(x, v) \in K \times \mathcal{V}, \\ f(x+t v, v) & \text { if } \quad(x+t v, v) \in K \times \mathcal{V}, \\ 0 & \text { elsewhere. }\end{cases}
$$

It is not hard to see that

$$
\begin{aligned}
\|g-h\|_{\omega} & =\int_{\tau_{t}(K \times \mathcal{V})} f(x+t v, v) \omega(x, v) d x d v \\
& =\int_{K \times \mathcal{V}} f(x, v) \omega(x-t v, v) d x d v \\
& \leq r(t)\|f\|_{\omega}
\end{aligned}
$$

which is sufficiently small for large enough $t$ and the same is true for $U_{0}(t) h-f$, since $\left\|U_{0}(t) h-g\right\|_{\omega} \leq r(-t)\|g\|_{\omega}$.

Let us denote by $U_{1}(t)$ the $C_{0}$-group generated by the penetration operator $T_{1}=$ $T_{0}+A_{a}$, where the absorption operator $A_{a}$ is defined by $A_{a} f=-\sigma_{a}(x, v) f$. The $C_{0}$-group $U_{1}(t)$ has an explicit form given by

$$
\left[U_{1}(t) f\right](x, v)=\exp \left\{-\int_{0}^{t} \sigma_{a}(x-s v, v) d s\right\} f(x-t v, v) .
$$

In this case the wave operators exist and have also the explicit forms 


$$
\begin{aligned}
W_{ \pm}\left(T_{0}, T_{1}\right) & =\lim _{t \rightarrow \pm \infty} e^{-\int_{0}^{t} \sigma_{a}(x-(s-t) v, v) d s} f(x, v) \\
& =e^{-\int_{\mp \infty}^{0} \sigma_{a}(x-\tau v, v) d \tau} f(x, v)
\end{aligned}
$$

and

$$
\begin{aligned}
W_{ \pm}\left(T_{1}, T_{0}\right) & =\lim _{t \rightarrow \pm \infty} e^{-\int_{0}^{-t} \sigma_{a}(x-\tau v, v) d \tau} f(x, v) \\
& =e^{-\int_{0}^{\mp \infty} \sigma_{a}(x-\tau v, v) d \tau} f(x, v) .
\end{aligned}
$$

Since $\sigma_{a}$ has a compact support with respect to $x$ in $\Omega$,

$$
\int_{-\infty}^{\infty} \sigma_{a}(x-t v, v) d t \leq v_{\min }^{-1} M_{a}[\operatorname{diam} \Omega]
$$

where $M_{a}=\left\|v^{-1} \sigma_{a}\right\|_{\infty}$ denotes the mean free path for $\sigma_{a}$. Thus, these wave operators are bounded in $L_{\omega}^{1}\left(\mathbb{R}^{n} \times \mathcal{V}\right)$ for any admissible weight function $\omega$. Here we assume the following:

$$
\inf _{(x, v) \in \mathbb{R}^{n} \times \mathcal{V}} \sigma_{a}(x, v)=\sigma>0 .
$$

Theorem 4.4. Assume (H5); then there exists a weight function $\omega$ satisfying the assumptions of Lemma 4.3 such that, in $L_{\omega}^{1}\left(\mathbb{R}^{n} \times \mathcal{V}\right)$, the $C_{0}$-group $U_{1}(t)$ is hypercyclic and satisfies

$$
\left\|U_{1}(t)\right\|_{\omega} \leq 1 \quad \text { for } \quad t \geq 0 .
$$

Proof. The hypercyclicity of $U_{1}(t)$ follows from the existence of the wave operators and Theorem 3.2. For $\alpha \leq \sigma$, we construct a weight function according to Lemma 4.3 ; then

$$
\begin{aligned}
\left\|U_{1}(t) f\right\|_{\omega} & =\int_{\mathbb{R}^{n} \times \mathcal{V}} e^{-\int_{0}^{t} \sigma_{a}(x-(s-t) v, v) d s}|f(x, v)| \omega(x+t v, v) d x d v \\
& \leq e^{(\alpha-\sigma) t}\|f\|_{\omega} \leq\|f\|_{\omega} \quad \text { for any } \quad t>0
\end{aligned}
$$

In spite of the contraction of $U_{1}(t)$ for $t \geq 0$, this group is not uniformly bounded for $t \leq 0$; hence we cannot use the Cook's Lemma (see [R-S]) for the existence of $W_{ \pm}\left(T, T_{1}\right)$ and $W_{ \pm}\left(T_{1}, T\right)$.

\section{REFERENCES}

[D-S-W] W. Desch, W. Schappacher and G.F. Webb, Hypercyclic and chaotic semigroups on linear operators, Ergodic Theory Dyn. Syst. 17 (1997), 793-819. CMP 97:17

[Em1] H. Emamirad, On the Lax and Phillips scattering theory for transport equation, J. Funct. Analysis 62 (1985), 276-303. MR 87c:47011

[Em2] Scattering theory for linearized Boltzmann equation, Transport Theory Statis. Phys. 16 (1987), 503-528. MR 89g:82087

[Em3] - Hypercyclicité du semi-groupe de transport et le théorème de représentation de Lax et Phillips, C. R. Acad. Sc. Paris 325 (1997), 157-162. CMP 97:17

[Em-A] H. Emamirad and P. Arianfar, Relationship Between Scattering and Albedo Operators in Computerized Tomography, Mathematical Population Dynamics (O. Arino et al., eds.), vol. 3, Wuerz Publishing Ltd (to appear).

[L-P1] P.D. Lax and R.S. Phillips, Scattering theory for transport phenomena, Proc. Conf. on Functional Analysis, Univ. California at Irvine, California, 1966. MR 36:3166

[L-P2] _ Scattering Theory, Academic Press, New York, 1967. MR 36:350

[Si] B. Simon, Existence of the scattering matrix for the linearized Boltzmann equation, Commun. Math. Phys. 41 (1975), 99-108. MR 53:4856 
[R-S] M. Reed and B. Simon, Methods of Modern Mathematical Physics. Vol.3, Academic Press, New York, 1979. MR 80m:81085

[Um] T. Umeda, Smooth perturbation in ordered Banach spaces and similarity for the linear transport operators, J. Math. Soc. Japan 38 (1986), 617-625. MR 87j:47017

Laboratoire de Modélisation Méchanique et de Mathématiques Appliquées, SP2Mi. Université de Poitiers, Boulevard 3, Teleport 2, BP 179. 86960 Futuroscope Cedex, FRANCE

E-mail address: emamirad@13ma.univ-poitiers.fr 